\title{
$\mathbb{T} \mathbb{E}$
}
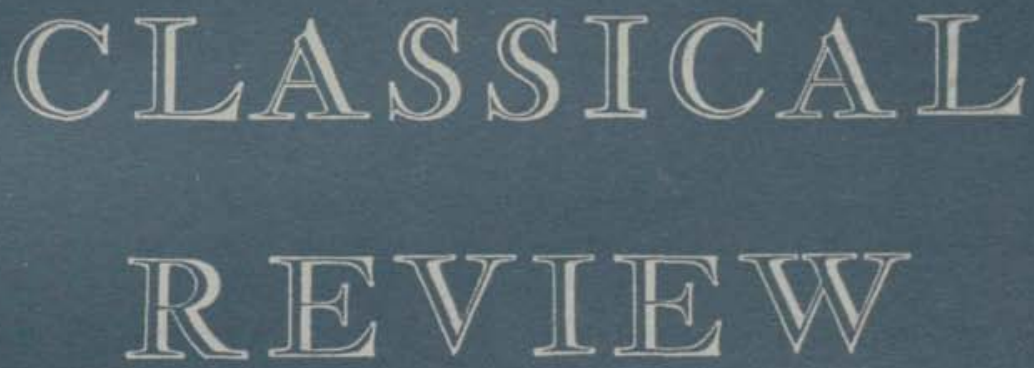

NEW SERIES VOLUME X NUMBER 2

(Vol. Lxxiv of the continuous series)

\section{Fune rg6o}

\section{OXFORD}

\section{AT THE GLARENDON PRESS}

LONDON NEW YORK TORONTO MELBOURNE 


\section{THE GLASSICAL REVIEW}

Editors C. J. FORDYCE, M.A., 3 The University, Glasgow, W. 2 R. M. RAtт n B U Y, M.A., Trinity College, Cambridge

All communications intended for the Editors should be sent to Mr. Fordyce. Books for review should be sent to the Editors, c/o The Clarendon Press, Oxford.

BOARD OF MANAGEMENT

Professor E. H. Warmington, M.A. (Chairman)

Professor R. G. Austin, M.A. (Hon. Treas.)

F. H. Sandbach, M.A.

Professor F. W. Walbank, M.A., F.B.A.

Professor W. S. Watt, M.A. (Hon. Sec.)

$\left\{\begin{array}{l}\text { Representing the } \\ \text { Council of the } \\ \text { Classical Association }\end{array}\right.$

A. G. Woodhead, M.A., representing the Cambridge Philological Society

M. Platnauer, B.Litt., M.A., representing the Oxford Philological Society With the co-operation of

Professor B. D. Meritt, Institute for Advanced Study, Princeton,

Professor J. A. FitzHerbert, University of Adelaide, and

Professor Homer A. Thompson, Institute for Advanced Study, Princeton
New Series

Volume $X$, No. 2
June 1960

\section{CONTENTS}

The Fate of Gessius

Notes on Cicero

Neglected Hyperbole in Juvenal

Aeschylus, Agamemnon 22-24

Herodotus vi. 72

Callimachus, Hymn vi. 88

A Homeric Parody in Lucian

Cicero, Philippics ii. 103

Horace, A.P. $37^{2-3}$

$\begin{array}{rr}\text { C. M. BOWRA } & 9 \mathrm{I} \\ \text { E. COURTNEY } & 95 \\ \text { E. L. HARRISON } & 99 \\ \text { D. S. ROBERTSON } & 102 \\ \text { M. PLATNAUER } & 102 \\ \text { K. J. MCKAY } & 102 \\ \text { M. D. MACLEOD } & \text { IO3 } \\ \text { R. G. M. NISBET } & \text { IO3 } \\ \text { J. G. GRIFEITHS } & \text { IO4 }\end{array}$

02

02

03

04

\section{REVIEWS:}

Der homerische Schiffskatalog und die Ilias (Jachmann), D. L. PAGE, 105; Homer and his Critics (Myres), J. A. DAvison, 108; Parmenide (Untersteiner), G. B. KERFERD, III; Vox Zenonis (Zafiropulo), G. B. KERFERD, I 12 ; Der Dialog Ion als Zeugnis platonischer Philosophie (Flashar), H. C. BALDRy, Ir3; Therapeia: Plato's Conception of Philosophy (Cushman), J. R. BAmbrovgh, I I5; Plato (Friedländer), J. R. BAmbrough, 117 ; Die Lehre von der Lust in den Ethiken des Aristoteles (Lieberg), G. B. Kerferd, I 18 ; Sexti Empirici Opera, i (Mutschmann-Mau), A. WAsserstern, r20; Dictionnaire historique de la terminologie gémetrique des greos (Mugler), I. Bulmer-Thomas, I 2 I ; Notes de lexicographie botanique grecque (André), D. E. ErcHнOLz, 123; Geschichte der griechischen Literatur (Lesky), H. LL. Hudson-wrurams, I24; Das Original des plautinischen Persa (Müller), G. w. Williams, 128; Ciceronis De Natura Deorum, ii, iii (Pease), M. L. Clarke, I30; Uber die klassische Theorie und Praxis des antiken Prosarhythmus (Schmid), A. E. Dovglas, I 3 I ; Patronus und Orator (Neuhauser), A. E. Dougl. As, 133; Ad Pyrrham (Storrs), L. P. WILkinson, 134; Horace's Ninth Epode (Wistrand), M. L. CL.ARKE, 136; Tibull (Helm), F. H. SANDBACH, 137; Rabirius: Bellum Actiacum (Garuti), E. J. KenNEx, 138; Lucans 


\title{
THE \\ C L A S S I C A L RE V I E W \\ sortre by \\ C. J. FORDYCE \\ AND \\ R. M. RATTENBURY
}

\author{
BOARD OF MANAGEMENT \\ Prop. E. H. WARMINGTON, M.A. (Chairman) \\ Pror. R. G. AUSTIN (Hon. Treasurer) \\ F. H. SANDBACH, M.A. \\ Prop. F. W. WALBANK, M.A., F.B.A. \\ Prop. W. S. WATT, M.A. (Hon. Secretary)
}

A. G. WOODHEAD, M.A. (Representing the Cambridge Philological Society)

M. PLATNAUER, B.LITT., M.A. (Representing the Oxford Philological Society)

With the co-operation of

Prof. B. D. MERITT, Institute of Advanced Study, Princeton

Pror. J. A. FITZHERBERT, University of Adelaide, and

Pror. HOMER A. THOMPSON, Institute of Advanced Study, Princeton

\author{
NEW SERIES \\ VOLUME X
}

(VOL. LXXIV OF THE CONTINUOUS SERIES)

Published in co-operation with the Classical Association OXFORD: AT THE CLARENDON PRESS I96o 
Oxford University Press, Amen House, London E.C.4 OLASOOW NEW YORK TORONTO MELBOURNB WELLINOTON BOMBAY CALCUTTA MADRAS KARAGEI KUALA LUMPUR CAPE TOWM IBADAN NAIROAS ACCRA

(C) Oxford University Press rg6o

\author{
The British Academy \\ made a generous contribution towards \\ the cost of this volume
}

PRINTED IN GREAT BRTTAN 


\title{
TABLE OF GONTENTS
}

\section{Number I}

Archilochus, fr. 2 Diehl

A Note on Fragment 12 of Anaxagoras

J. A. DAVISON I

Horace, Odes iv. 15. 29

Sophocles, O. T. 230-2

Plato, Phaedo $84 \mathrm{c}$

The Ghost of a Longaevus Parens

Claudian, De Raptu Proserpinae, ii. 163-9

The Alleged Aetolian Embassy to Rome

\author{
A. WASSERSTEIN 4 \\ R. D. WILLIAMS 6 \\ N. B. BOOTH 7 \\ W. L. LORIMER 7 \\ J. H. BISHOP 8 \\ H. H. HUXLEY 8 \\ G. A. DOREY 9
}

\section{REVIEWS :}

Archiloque, Les Fragments (Lasserre-Bonnard), K. J. DOVER, 10; Les Scholies métriques de Pindare (Irigoin), D. s. ROBERTson, 12; Pitagorici, testimonianze e frammenti (Timpanaro Cardini), G. B. RERFERD, 15; Sophocle, Ajax, Oedipe Roi, Electre (Dain-Mazon), A. M. DALE, I6; A Study of Sophoclean Drama (Kirkwood), D. W. LuCAs, 19; Sophocles the Playuright (Adams), P. T. STEVENs, 21 ; Sophocles-Dramatist and Philosopher (Kitto), P. T. STEvens, 23; Ein neues InachosFragment des Sophokles (Pfeiffer), H. LLOYD-JONBs, 25; The Poetry of Greek Tragedy (Lattimore), H. C. BALDRY, 26; Beobachturgen zu einigen 'recentiores' des Thukydides (Kleinlogel), K. J. DOVER, 28; Recherches sur la Tradition Platonicienne (Courcelle and others), R. Bambrough, 29; Der Panegyrikos des Isokrates (Buchner), H. LL. HuDson-WTLLIAMs, 31 ; Excadations at $\mathcal{N}$ essana, iii (Kraemer), H. I. BelL, 33; Roman Literary Portraits (Löfstedt), w. s. maguinness, 35; Cicero, The Speeches (Gardner), R. G. M. NISBET, 37; Pline l'Ancien, Histoire Naturelle, xiv (André), D. E. EIcHHOLz, 39; Dictys Cretensis Ephemeridos Belli Troiani libri (Eisenhut), R. BRownIng, 40; Avianus, Fabulae (Guaglianone), R. BRownING, 42; The Latin Josephus, i (Blatt), R. BROWNING, 44; Introduction à l'etude de la versification latine médiévale (Norberg), R. BRownING, 46; Gli eroi greci (Brelich), H. J. ROsE, 48; Moral Values in the Ancient World (Ferguson), A. W. H. ADKINs, 50; Abhängige Orte im griechischen Altertum (Gschnitzer), N. G. L. HAMMOND, 52; Essays in Greek History (WadeGery), H. D. WEstlake, 54; La lega tessala fino ad Alessandro Magno (Sordi), H. D. WEstLAKE, 55; The Generalship of Alexander the Great (Fuller), G. L. CAwkwell, 57; La grecita politica da 7 ucidide ad Aristotele (Pavan), T. A. sINclaIR, 59; Die Griechische Landschaften, ii. 2 (Philippson-Kirsten), N. G. L. HAMMOND, 60; Römische Geschichte, iii (Altheim), E. S. STAVELEY, 62; Album of Dated Latin Inscriptions, i (Gordon-Gordon), Contributions to the Paleography of Latin Inscriptions (GordonGordon), J. M. REYNolds, 64; Le Patronat sur les Collectivités Publiques des origines au Bas-Empire (Harmand), w. K. LACEY, 66; Cleopatra: A Study in Politics and Propaganda (Volkmann), J. P. V. D. BALSDON, 68; Les Trophées romains (Picard), J. M. c. ToYNBeE, 71 ; Grain-Mills and Flour in Classical Antiquity (Moritz), D. E. EIchrolz, 73; Pagan Mysteries in the Renaissance (Wind), R. R. BOLGAR, 75 .

Short Reviews

Summaries of Periodicals $\quad \mathbf{8}_{3}$

Notes and News

Books Received

Number 2

The Fate of Gessius

C. M. BOWRA

Notes on Cicero

E. GOURTNEY

Neglected Hyperbole in Juvenal

E. L. HARRISON

Aeschylus, Agamemnon 22-24

D. S. ROBERTSON IO2

Herodotus vi. 72

M. PLATNAUER IO2

Callimachus, Hymn vi. 88

K. J. MAGKAY 103

A Homeric Parody in Lucian

M. D. MAGLEOD IO

Cicero, Philippics ii. 103

R. G. M. NISBET IO 3

Horace, A.P. 372-3

J. G. GRIFFITHS IO4

\section{REVIEWS :}

Der homerische Schiffskatalog und die Ilias (Jachmann), D. L. PAGE, 105; Homer and his Critics (Myres), J. A. Davison, 108 ; Parmenide (Untersteiner), g. B. Kerferd, I I I Vox Zenonis (Zafiropulo), G. B. KERFERD, 112 ; Der Dialog Ion als Zeugnis platonischer Philosophie (Flashar), H. c. 
Plato (Friedländer), J. R. BAMBrough, I I 7; Aristotle's Doctrine of Pleasure (Lieburg), G. B. KERFERD, 118 ; Sexti Empirici Opera, i (Mutschmann-Mau), A. WASSERSTEIN, 120; Dictionnaire historique de la terminologie geometrique des grecs (Mugler), I. BULMER-THOMAs, 121 ; Notes de lexicographie botanique grecque (André), D. E. EIGHHOLz, 123; Geschichte der griechischen Lileratur (Lesky), H. LL. hudson-williams, 124; Das Original des plautinischen Persa (Müller), g. w. williams, 128; Ciceronis De Natura Deorum, ii, iii (Pease), M. L. CLARKE, I30; Uber die klassische Theorie und Praxis des antiken Prosarhythmus (Schmid), A. E. Douglas, 13I ; Patronus und Orator (Neuhauser), A. E. Douglas, 133; Ad Pyrrham (Storrs), L. P. wILkinson, 134; Horace's Ninth Epode (Wistrand), M. L. ClaRKe, I36; Tibull (Helm), F. H. SANDBAGH, I37; Rabirius: Bellum Actiacum (Garuti), E. J. KENNEY, 138; Lucans Gedicht vom Bürgerkrieg (Syndikus), E. J. KENNEY, 139; Griechische Sprachwissenschaft, ii (Brandenstein), D. M. JONEs, I40; Anredeformen (Svennung), D. M. JONES, I42; The Tongues of Italy (Pulgram), D. M. JONEs, 143 ; Notions fondamentales de la pensée religiense dans la Grèce classique (Rudhardt), w. K. c. GUTHRIE, I45; Le culte de Cérés (Le Bonniec), M. J. BOYD, 147; Histoire et historiens dans l'antiquité (Entretiens Hardt), J. P. v. D. BALSDON, I5I; Minoica (Sundwall Festschrift), D. M. JONES, 154 ; Solone (Masaracchia), A. R. W. HARRIsON, I56; Die Friedenspolitik des Perikles (Dienelt), P. A. BRUNT, I57; Epaminonda (Fortina), H. D. WEstLAKe, I59; L'Italia agraria sotto Traiano (Sirago), P. J. CUFF, I6I ; L'Autonomisme et christianisme dans l'Afrique romaine (Brisson), w. H. C. FREND, 162; Roman and Native in North Britain (Richmond), J. M. C. TOYNBEE, I64; Classical Education in Britain, 1500-1900 (Clarke), D. c. C. YouNG, 166.

Short Reviews

168

Summaries of Periodicals

Notes and News

Books Received

\section{Number 3}

The Olympic Festival of 324 B.c.

B. R. SEALEY

Aquilae Senectus

G. B. TOWNEND 186

A Gerundive in Juvenal

An unnoticed fragment of Sappho?

Parmenides, fr. 6

The transformation of Io, Ox. Pap. xxiii, 2369

Tacitus, Annals xv. 44. 3-8

J. G. GRIFFITH

189

R. BROWNING I92

M. C. STOKES I93

A. M. DALE I 94

K. F. C. ROSE I95

REVIEWS :

Lexikon des frühgriechischen Epos (Snell, Fleischer, Mette), J. A. DAvison, I95; Daily Life in the Time of Homer (Mireaux), D. H. F. GRAY, I97; Bühnenbearbeitung äschyleischer Tragödien (Böhme), D. w. LucAs, I98; Sofocle (Maddalena), D. w. Lucas, 200; The Fragments of Attic Comedy, ii (Edmonds), D. MERVYN JONEs, 202; Menander, Dyscolos (Bingen), Menandro: Dyskolos (Diano), Note in margine al Dyskolos (Diano), Menandros, Dyskolos (Mette), Notes on the Dyscolos (Quincey, Ritchie, Shipp, Treweek), The Birth of Modern Comedy (Webster), F. H. SANDBach, 204; An Introduction to the Greek Theatre (Arnott), D. W. LuCAS, 207; Hellenica Oxyrhynchia (Bartoletti), H. D. Westrake, 209; Die Schule des Aristoteles, x (Wehrli), G. B. KERFERD, 2 I ; Damascius, Lectures on the Philebus (Westerink) A. WASSERSTEIN, 212 ; Plutarch, Moralia, vol. vii (DeLacy and Einarson), F. H. SANDBACH, 2 I4; A Catalogue of the Greek Papyri in the Collection of Wilfred Merton (Rees, Bell, Barnes), E. G. TURNER, 215 ; Pauli Alexandrini E'oaywriká (Boer), I. BULMER-THOMAs,

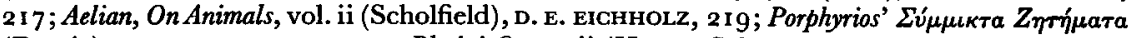
(Dörrie), A. H. ARMSTrong, 220; Plotini Opera, ii (Henry, Schwyzer), A. H. ARMstrong, 221 ; Atti del Convegno Internazionale Ovidiano, 1958, E. J. KENNEY, 222; The Latin Love Elegy (Luck), E. J. KENNEY, 224; Caesaris Commentarii de Bello Civili (Kraner, Hofmann, Meusel), J. H. simon, 226; Cicéron, Traité des Lois (de Plinval), R. G. M. NISBET, 228; Livy, vol. xiv (Schlesinger), A. H. Modonald, 229; Tacite, Histoires, $i$ (Wuilleumier), r. H. Martin, 230; Tacitus, Annals, $i$ (Miller), R. H. MARTIN, 232; Tacitus, Germania (Fehrle, Hünnerkopf), R. H. MARTiN, 234; Late Latin (Löfstedt), R. BRowning, 235; On Translation (Brower), T. F. Higham, 237; Studies in the Greek Negatives (Moorhouse), Negative Conditional Sentences in Greek (Koppers), K. J. Dover, 24I ; Die Gliederung des indogermanischen Sprachgebiets (Porzig), A. J. BEATTIE, 243; Lydiaka (Heubeck), D. M. JONES, 245; From the Gracchi to Nero (Scullard), A. H. McDONALd, 247; Hellenistic Science (Sarton), A Source Book in Greek Science (Cohen and Drabkin), D. E. EIchHolz, 250; Some School Books, B. H. KEMBALL-COOK, 252.

Short Reviews 